\section{Informations juridiques complémentaires sur la révision des dispositions pénales applicables à la corruption}

HP. Kuhn, avocat, secrétaire général adjoint FMH

L'article explique la toile de fond juridique de l'avis d'expert de la commission AQ de la Société Suisse de Gynécologie et d'Obstétrique: quel est le texte déterminant du code pénal révisé? Comment doit-il être interprété sur la base du message du Conseil fédéral? Qu'est-ce qui est similaire à l'Allemagne, qu'est-ce qui en diffère?

\section{Texte de loi du code pénal révisé}

Art. $322^{\text {quater }}$ Corruption passive:

"Celui qui, en tant que [...] fonctionnaire, [...], aura sollicité, se sera fait promettre ou aura accepté un avantage indu, en sa faveur ou en celle d'un tiers, pour l'exécution ou l'omission d'un acte en relation avec son activité officielle et qui soit contraire à ses devoirs ou dépende de son pouvoir d'appréciation sera puni de la réclusion pour cinq ans ou plus ou de l'emprisonnement."

Art. $322^{\text {sexies }}$ Acceptation d'un avantage:

"Celui qui, en tant que [...] fonctionnaire, [...], aura sollicité, se sera fait promettre ou aura accepté un avantage indu pour accomplir les devoirs de sa charge sera puni de l'emprisonnement ou de l'amende." Art. $322^{\text {octies }}$ Dispositions communes:

"1. Si la culpabilité de l'auteur et les conséquences de son acte sont si peu importantes qu'une peine serait inappropriée, l'autorité compétente renoncera à le poursuivre, à le renvoyer devant le tribunal ou à lui infliger une peine.

2. Ne constituent pas des avantages indus les avantages autorisés par le règlement de service et ceux qui, de faible importance, sont conformes aux usages sociaux.

3. Les particuliers qui accomplissent des tâches publiques sont assimilés aux agents publics."

Définir, au niveau interne à l'hôpital, les "cadeaux insignifiants et admis socialement»

Le droit pénal révisé permet (et exige en même temps!) l'élaboration de règlements internes clairs, pour les hôpitaux et les facultés, en vue de définir ce que sont les cadeaux insignifiants et admis socialement. Le Conseil fédéral a déclaré à ce sujet dans le message: "Un avantage est indus lorsque l'agent public qui devrait en bénéficier n'a pas le droit de l'accepter. Cette définition permet d'exclure notamment du champ de l'infraction de corruption les libéralités dont l'acceptation est permise par les règlements de service. On songera par exemple à un texte administratif régissant l'annonce, la faculté d'accepter ou, au contraire, l'obligation de remettre les dons et autres avantages reçus. Mais l'exigence que l'avantage soit indu donne également une certaine latitude pour exclure de l'infraction les libéralités insignifiantes et admises socialement." ${ }^{1}$

Pour les médicaments, le Parlement a du reste intégré dans la nouvelle loi sur les produits thérapeutiques, qui devrait entrer en vigueur au $1^{\text {er }}$ janvier 2002, le règlement de l'OICM sur les dotations de faible valeur autorisées sans y effectuer de modifications: "Art. 33 Loi sur les produits thérapeutiques, Promesse et acceptation d'avantages matériels

[...]

2 Il est interdit aux personnes qui prescrivent ou qui remettent des médicaments ainsi qu'aux organisations qui emploient de telles personnes de solliciter ou d'accepter des avantages matériels.

3 Sont admis:

a. les avantages matériels de valeur modeste et qui ont un rapport avec la pratique de la médecine ou de la pharmacie;

b. les rabais usuels dans le commerce et justifiés économiquement qui se répercutent directement sur le prix."

Il y a également lieu de définir, dans des directives internes aux hôpitaux, quels cadeaux de patients peuvent être acceptés par les médecins et le personnel de soin, et si ces cadeaux doivent, à partir d'une certaine valeur, être déclarés ou même soumis à autorisation.

\section{Financement externe de la recherche - souhaité, mais: créer la transparence, ne pas accepter les libéralités à des personnes individuelles}

Dans le message relatif à la révision du code pénal, le Conseil fédéral s'exprime explicitement sur cette question: ${ }^{2}$ "Il est désormais courant que, dans le cadre d'un financement externe ou de sponsoring, des institutions de droit public fassent appel au secteur privé pour financer volontairement des tâches dévolues à l'Etat. Il est évident que dans le financement externe et le sponsoring d'activités étatiques, le but recherché est tout autre que la corruption: il s'agit en effet d'une prise en charge ouvertement déclarée, parfaitement licite, responsable et volontaire de certaines tâches publiques par des particuliers.»³

Et de poursuivre: "Mais on ne saurait exclure que la corruption prenne le visage d'un financement externe. Lorsqu'un médecin chef de clinique touche, à titre privé, une participation aux bénéfices de l'en- 
treprise qu'il a favorisée comme fournisseur de médicaments pour son établissement hospitalier, les termes de financement externe ou de sponsoring sont là pour enjoliver une réalité qui s'appelle corruption." ${ }^{4}$

Ce qui fait la différence entre un financement externe souhaité et un sponsoring, d'une part, et la corruption, d'autre part, est la question de savoir qui reçoit les fonds: "Mais dans la plupart des cas, cette distinction est opérée par le seul fait que le financement est accordé à une institution, c'est-à-dire un vrai tiers, et non pas à des particuliers. Contrairement à la corruption proprement dite, l'octroi d'un avantage à des tiers ou l'acceptation d'un avantage par des tiers n'est pas punissable..1 ${ }^{5}$

\section{Commentaire}

Il existe ici une nette différence par rapport à certaines décisions des tribunaux allemands. En Allemagne, par exemple, un médecin a été condamné à cause d'appareils techniques médicaux qui ont été mis gratuitement non pas à sa disposition personnelle, mais à la disposition de l'hôpital public. ${ }^{6}$ De telles décisions sont contestées en Allemagne même et ont déclenché une certaine perplexité chez les médecins et les hôpitaux - cela n'est pas à craindre en Suisse en raison des différences de règlements légaux.

Concernant l'importance centrale de la transparence nécessaire, citons le Conseil fédéral: «Les autres contributions ou libéralités octroyées à titre personnel peuvent être éliminées sans difficulté en recourant à une interprétation appropriée de l'exigence de l'avantage indu. Dans ce domaine, précisément, la publicité et la transparence constituent un critère essentiel. Celui qui fournit des fonds sans aucune arrière-pensée de corruption ne craindra pas que le fait soit publié et que ses concurrents en prennent connaissance. $D u$ côté du destinataire, on pensera notamment aux communications faites à l'autorité supérieure compétente. Quant aux autres cas limite, ils pourront être résolus par le biais de l'art. $322^{\text {octies }}$ ch. 1 [du projet - remarque de l'auteur] P-CP.» ${ }^{7}$

\section{Commentaire}

$\mathrm{Au}$ Parlement, les propos du Conseil fédéral cités concernant le financement de la recherche et la transparence ont été acceptés sans commentaire aussi bien dans l'assemblée plénière du Conseil national et des Etats qu'au sein des commissions consultatives. Le message du Conseil fédéral reflète ainsi de manière coercitive l'intention du législateur.

En ce qui concerne la transparence, c'est-à-dire la déclaration et, le cas échéant, l'autorisation d'un financement externe et du sponsoring, la situation juridique suisse est comparable à celle de l'Allemagne. ${ }^{8-11}$

Du reste: la recherche à financement externe est aussi clairement souhaitée par la nouvelle loi fédérale sur l'aide aux universités. Car la détermination des subventions fédérales aux universités a égale- ment lieu, sur la base de l'art. 15, para. 3, de la loi fédérale sur l'aide aux universités, selon le critère «Prestations de recherche et l'acquisition de fonds de tiers (fonds issu du Fonds national, des projets de l'UE, des CTI ainsi que de fonds de tiers privés et autres fonds de tiers publics)».

\section{Définitions dans le nouveau code pénal: "Fonctionnaire", "Avantages déterminants"}

Les "fonctionnaires" qui se laissent corrompre sont passibles d'une peine. Il peut p. ex. s'agir d'un médecin dans un hôpital public ou d'un chargé de cours dans une faculté de médecine. Le code pénal ne mentionne en revanche pas la corruptibilité du secteur privé $^{12}$ (cabinet privé, médecin agréé dans un hôpital privé). Pour délimitation: d'après le Conseil fédéral, «il est indifférent de savoir sous quel statut juridique une personne accomplit des tâches au service de la collectivité; ce qui est déterminant, c'est qu'elle accomplisse des tâches dévolues à l'Etat.» ${ }^{13}$ Cela signifie qu'il est indifférent que le médecin d'un hôpital public ait le statut de fonctionnaire, d'emploi de droit public ou d'employé de droit privé, ou qu'il s'agisse d'un médecin agréé autorisé par contrat. C'est la fonction exercée dans le service public qui est déterminante.

"Avantage" au sens du code pénal signifie "toute amélioration objectivement mesurable - juridique, économique ou personnelle». ${ }^{14}$

Dans l'article sur la corruption, il s'agit des avantages "destinés à influencer l'agent public» ${ }^{15}$ c'est-àdire d'actes futurs de l'agent public. Dans son message, le Conseil fédéral explique cela sur la base d'un exemple issu de l'hôpital: "Le bouquet de fleurs offert à l'infirmière [...] a pour fonction première de remercier l'intéressée pour les bons services rendus dans le passé et non pas d'influencer l'accomplissement ultérieur des devoirs liés à la charge. Même indépendamment de la question de savoir si l'avantage est (indu, il manque ici le caractère répréhensible.»" ${ }^{16}$

\section{Les honoraires adaptés à la prestation sont permis}

Cette question a été clarifiée par le Conseil fédéral à l'exemple de cours de rattrapage: «En revanche, l'institutrice qui, prenant sur son temps libre, se fait payer pour donner des cours de rattrapage à des élèves en difficulté ne reçoit pas une récompense spour qu'elle accomplisse les devoirs de sa charge', aussi longtemps du moins que le prix demandé reste dans un rapport adéquat avec la prestation fournie.» ${ }^{17}$

\section{Commentaire}

Ce que le Conseil fédéral déclare concernant l'institutrice vaut de manière analogue tout aussi bien pour le traitement privé par le médecin en chef ou le médecin agréé dans un hôpital public: sans importance tant que les honoraires sont adéquats. 


\section{Concernant la différence entre la "corruptibilité» et la simple "acceptation d'un avantage»}

"[...] la corruption proprement dite continuera de supposer un rapport avec une action ou une omission au moins déterminable.» Sinon, il n'y aura pas corruption mais simplement l'acceptation d'un avantage, passible d'une peine plus légère (ce que l'on appelle "l'alimentation progressive»). ${ }^{18}$

La "violation des devoirs de la charge» dans la corruption comporte, d'après le Conseil fédéral, aussi bien les actes accomplis par l'agent public «dans le cadre de ses compétences" que ceux dont il n'a pas "la compétence» mais qu'il a seulement la "possibilité d'accomplir en raison de sa position officielle». Les "actes purement privés", en revanche, en sont exclus. ${ }^{19}$

Message Feuille fédérale 1999, p. 5076.

2 Message ch. 213.4, Feuille fédérale 1999, p. 5085-6.

3 Message Feuille fédérale 1999, p. 5085-6.

4 Message, Feuille fédérale 1999, p. 5085.

Message Feuille fédérale 1999, p. 5086.

6 En Suisse, on ne décidera donc pas comme on le ferait en Allemagne (soulevant de nombreuses contestations): "Einer Strafbarkeit [...] steht nicht entgegen, dass gerade die Anstellungskörperschaft des mittelbar besser gestellten Amtsträgers eine objektiv messbare Besserstellung erfuhr." Décision du Oberlandesgericht de Karlsruhe du 30.3.2000 - 2 Ws 181/99; communiqué dans la MedR 10/2000; 485-6. Commentaire du rapporteur allemand: «Les médecins et les institutions médicales sont laissés seuls avec cette décision: d'une part, la réforme imminente des dispositions juridiques en matière de service pour les enseignants des hautes écoles encourage l'acquisition de fonds issus de l'industrie et rend nécessaire la réduction des budgets de la recherche. D'autre part, l'autorisation par le comité de la clinique ou la direction de la haute école n'aurait même pas aidé l'accusé."

7 Message ch. 213.4., Feuille fédérale 1999, p. 5086.

8 A ce sujet, il y a eu en Allemagne des cas devant nécessairement aboutir à une condamnation et qui auraient la même issue en Suisse, par exemple lorsque le médecin d'un hôpital public était, d'une part, responsable des commandes et, d'autre part, «bénéficiait d'avantages dans sa vie privée» (citation extraite des commentaires de Jens Göben sur le jugement de la cour fédérale de Justice du 19.10.1999 - 1 StrR 264/99, publiés dans MedR 4/2000; 193-5).

9 Concernant la situation dans le dit "complexe de la valvule du cœur»: à l'origine, une enquête a été menée contre 2700 chirurgiens cardiologues et 460 hôpitaux. Les investigations ont débouché deux ans plus tard sur un "procès contre 132 collaborateurs des entreprises de livraison et 1501 médecins dans 390 hôpitaux. [...] Entre-temps, 1059 procédures ont été suspendues sans obligations (70 pour-cent), parce que les conditions d'une mise en accusation après le soupçon initial faisaient défaut. [...] Sont restées 442 procédures contre des médecins; 200 d'entre elles sont clôturées aujourd'hui. 32 cas ont fait l'objet d'une mise en accusation. Ce n'est que dans moins de dix cas qu'un jugement exécutoire a été prononcé, avec amende, généralement dans des procédures contre des non-médecins." (Harald Clade, Deutsches Ärzteblatt 44/2000 du 3.11.2000; 2189)

\section{Commentaire}

Est donc "corruptible» celui qui, en tant que fonctionnaire, accepte un avantage indu dans le but de se montrer reconnaissant, en contrepartie, vis-à-vis du donateur, donc de réagir par un «acte impliquant une violation des devoirs de sa charge». On est en revanche en présence de la simple acceptation d'un avantage, passible d'une peine plus légère, lorsqu'un avantage indu est certes accepté, mais que le bénéficiaire ne s'en montre pas reconnaissant et n'a pas non plus l'intention de le faire ultérieurement.

\section{Deutsch erschienen in Nr. 14/2001}

La traduction de cet article a été possible grâce à la participation financière de la maison Novartis Pharma Schweiz AG, Berne, que nous remercions.

10 «Das Bemühen um eine hinreichende Trennung von Zuwendung und Beschaffung sowie um die Transparenz des Mittelflusses" (Les efforts déployés pour obtenir une séparation suffisante entre libéralité et acquisition ainsi que pour assurer la transparence de la liquidité) [commentaire de Jens Göben sur la décision de la cour fédérale de Justice du 19.10.1999; publié dans MedR 4/2000; 193-5; 194] sont les indices d'un comportement correct et contre la corruptibilité et l'acceptation d'avantages indus.

1 Voir également la décision du Oberlandesgericht du 14.1.2000 2 Ws 243/99, publiée dans MedR 8/2000; 371-6: il s'agissait d'un contrat de conseil entre un médecin en chef (à l'époque) et l'industrie de la technique médicale. L'activité annexe du médecin en tant que telle avait été autorisée. En même temps, l'hôpital entretenait une relation commerciale avec l'entreprise conseillée: il acquérait auprès de ce constructeur des stimulateurs cardiaques, et la décision d'acquisition n'avait certes pas été prise de droit, mais de fait par ce médecin en chef. Tel que le cas se présentait devant le tribunal, ce constructeur de stimulateurs cardiaques avait comme politique systématique d'octroyer des contributions aux "voyages et aux participations aux congrès, aux honoraires pour les études, aux documentations et aux séminaires ainsi qu'à la conclusion d'accords de base orientés sur le chiffre d'affaires en y associant les achats de produits médicaux pouvant être attendus pour le présent et pour l'avenir.» Ce qui a été déterminant pour le tribunal étaient les efforts déployés par le médecin pour "dissimuler" à son hôpital "la véritable étendue de son activité de conseil» pour cette entreprise.

Dans quelles circonstances l'autorisation du contrat de conseil et de paiements par l'hôpital aurait-elle été juridiquement valable? Dans le récapitulatif, les rédacteurs s'entendent à affirmer que ce qui est déterminant dans une telle situation, c'est que «le rapport avec la promesse et/ou l'acceptation de l'avantage soit déclaré ouvertement à l'autorité responsable de l'autorisation".

12 En 1999, le Conseil fédéral a, dans son projet de loi, écarté le thème de la corruption privée pour des raisons de temps, parce que d'autres questions s'imposaient en partie (message 19.4.1999, Feuille fédérale 1999, p. 5045-102, 5068). Le Parlement s'est rallié au Conseil fédéral.

13 Message Feuille fédérale 1999, p. 5073.

${ }^{14}$ Message Feuille fédérale 1999, p. 5075.

${ }_{15}$ Message Feuille fédérale 1999, p. 5084.

${ }_{16}$ Message Feuille fédérale 1999, p. 5084.

17 Message Feuille fédérale 1999, p. 5084.

${ }_{18}$ Message Feuille fédérale 1999, p. 5082.

19 Tout dans le message, Feuille fédérale 1999, p. 5078. 